\title{
Congestion Control for Biological Nanoscale Cyber-Physical Systems
}

\author{
Invited Paper \\ Luca Felicetti, Mauro Femminella and Gianluca Reali \\ Dept. of Engineering, University of Perugia, CNIT RU \\ via G. Duranti 93, 06125 Perugia, Italy \\ ing.luca.felicetti@gmail.com, mauro.femminella@unipg.it, gianluca.reali@unipg.it
}

\begin{abstract}
Cyberphysical systems (CPSs) are a new class of engineered systems that offer close interaction between cyber and physical components, by integrating three main components: communications, control, and computing. When these systems are brought to the nanoscale, some design and implementation issues arise. A further level of complexity is due to the use of biological components in a CPS, such as engineered cells, which may play the role of sensors, actuators, or even controller. In this paper we study the effectiveness of control solutions implemented through the usage of molecular communications in a biological nanoscale cyber-physical system (BioNanoCPS), where a biological nanomachine plays the role of actuator, that releases drug molecules, and another has the role of both sensor and controller. The goal of the proposal is to control the release rate, so that target cells can receive the desired amount of drug in a given time, by limiting potential side effects. Basically, we aim to limit congestion, which can arise when large amounts of molecules are released towards a target. To this aim, we propose a simple congestion detection scheme, and compare different rate control algorithms used to throttle the molecules release rate at the transmitter upon the reception of a feedback signal sent by the receiver. We validate the proposed techniques against delivery efficiency and delivery time of molecules by means of an extensive simulation campaign.
\end{abstract}

\section{Categories and Subject Descriptors}

I.6.3 [Simulation and Modeling]: Model Validation and Analysis

\section{Keywords}

Congestion detection, congestion control, feedback-based rate control, diffusion-based molecular communications.

\section{INTRODUCTION}

Cyber-physical systems (CPSs) are a new class of engineered systems that include close interaction between cyber and physical components, by integrating three main components: communica-

Permission to make digital or hard copies of all or part of this work for personal or classroom use is granted without fee provided that copies are not made or distributed for profit or commercial advantage and that copies bear this notice and the full citation on the first page. To copy otherwise, to republish, to post on servers or to redistribute to lists, requires prior specific permission and/or a fee.

BODYNETS 2016, December 15-16, Turin, Italy

Copyright @ 2017 EAI 978-1-63190-132-4 tions, control, and computing [1]. CPSs are expected to play a major role in the research, design, and implementation of future technologies. When these systems involve devices at the nanoscale, some design and implementation issues arise. A further level of complexity is brought by the use of biological components in a CPS, such as engineered cells, which may play the role of sensors, actuators, or even controllers in living bodies. Such systems are emerging as natural exploitation of the field of molecular communications [2-4], in which artificial nanomachines and/or engineered cells communicate by means of the exchange of molecules, by mimicking the natural cell signaling. Molecular communication is a novel paradigm of communication happening between biological nanomachines (specifically bio-nanomachines) over short ranges, within aqueous environments. In this model, a transmitter nanomachine emits molecules, which act as communication signals, and are received by receiver nanomachines. The reception process usually consists of a chemical reaction between signal molecules (ligand) and compliant receptors present on the receiver surface. Given the intrinsic bio-compatibility of these systems, they could allow implementing closed loop systems (which make them CPS), which offer the possibility to tightly monitor and control biological processes. These features are expected to give birth to really disruptive applications in the fields of medicine, food production, and so on.

In this paper, we propose to use a biological, nanoscale cyberphysical system (BioNanoCPS) to control the process of drug delivery/tissue engineering [5-7] directly in situ. We assume that a number of biological nanomachines (bio-nanomachines) play the role of actuators, that are able to release drug molecules, and another bio-nanomachine has the role of both sensor and controller. When the controller detects the proximity of a given target (e.g. a tumor), it triggers the release of drugs from actuators through a command sent by using molecular communications. However, since the distance between actuators and and target is not known in advance, the release rate has to be adjusted in order to avoid wasting of molecules. Thus, the first tasks of the controller is to monitor the drug absorption rate (sensor function), by absorbing the emitted drugs as if it were the receiver. The second one is to control the drug release rate. The goals of this second function are both to allow target cells receiving the desired amount of drug in a given time and, at the same time, to avoid releasing an amount of drugs that cannot be absorbed by the target, which could likely produce undesired side effects and drug waste. Even the messages used to control the release rate are sent by using molecular communications.

In this paper, we focus on the effectiveness of the control solutions implemented through the usage of molecular communications in a BioNanoCPS. In more detail, we aim to limit the phenomenon of congestion due to accumulation of drug molecules, already in- 
troduced in [8] and further elaborated in [9], which can arise when a large amount of molecules are released towards a target. To this aim, we propose both a simple congestion detection scheme (first contribution), which can be implemented in a bio-nanomachine, and four different rate control algorithms (second contribution), all based on the negative feedback concept [10]. We compare these different rate control algorithms used to throttle the molecules release rate at the transmitter upon the reception of a feedback signal sent by the receiver. Some of them inspire to control mechanisms implemented in the TCP protocol, mentioned in [11]. Without any loss of generality, we use the communication protocols already defined in [11], which allows focusing only on the effectiveness of control algorithms. We have assessed the performance of the proposed techniques against delivery efficiency and delivery time of molecules. Performance have been estimated by means of an extensive simulation campaign, implemented by using the BiNS2 simulator $[12,13]$.

The paper is organized as follows. In section 2, we illustrate the related work in the field. Section 3 presents the system model. In section 4, we illustrate our congestion detection scheme. Section 5 shows our proposal for control actions to be implemented in the TX node, and the relevant decision logic to be implemented in the RX node. The results of the simulation campaign, used to validate the proposed mechanisms and to analyze the trade-off between throughput and communication efficiency, are presented in Section 6. Finally, we draw our conclusions in Section 7.

\section{BACKGROUND AND RELATED WORKS}

Major efforts in the area of molecular communications are focused on the physical layer issues of various types of molecular communication media. Information capacity and physical features (e.g., delay, signal attenuation, amplification, and energy requirements) of molecular communications are typically studied by using random walk models [14-16], random walk models with drift $[17,18]$, diffusion-reaction-based models $[19,20]$, active transport models [14,21], collision-based models [22], and diffusion-based models [23-27], which is used in this paper.

Most of results have been obtained by assuming that a sufficiently high density of receptors are deployed on the receiver surface, so that all the available molecules coming in contact with the receiver surface come also in contact with the receptors and are absorbed (absorbing receiver assumption, [28]). A more refined model, which makes use of a finite number of receptors with a specific size, is presented in [29]. Although this model is more realistic, it assumes that each time a ligand molecule comes in contact with a compliant receptor, it is immediately absorbed (absorbing receptor assumption). Hence, the stochastic nature of ligand-receptor binding is not considered. Differently, a finite bonding duration is analyzed in [30], and further investigated in [31], which takes also into account the trafficking time [32], which can be essentially regarded as the molecule reception time. Specifically, it is defined as the time duration since the formation of the bond between a ligand molecule and a compliant receptor to the time instant in which, after the complex ligand-receptor has been internalized, the receiver is able to expose another free receptor on its surface. A reversible receiver model, which does not explicitly model receptors but rather their macroscopic behavior by means of a reaction constant, is presented in [33].

Finally, the papers $[8,9]$ analyze the congestion phenomenon in molecular communications, and provide theoretical models to predict it when a continuous stream of drugs are released towards a target. In this work, starting from these results, we develop a detection scheme able to early identify congestion conditions when a flow of molecules is released towards a target with variable rate and unknown release distance.

\section{THE SYSTEM MODEL}

The considered communication scenario consists of two fixed bio-nanomachines at a distance $d$ : one acting as receiver and control node (RX), and the other as transmitting node (TX). Both TX and $\mathrm{RX}$ are assumed to be spherical nanomachines, with radius $r_{T X}$ and $r_{R X}$, respectively. This is a simplified model, since it does not explicitly consider the effect of the target (e.g. tumor cells) on the sensing process carried out by the RX. However, an estimation of the presence of additional receivers (i.e. target cells) can be obtained by using the results presented in [34]. The communication happens by using two different types of molecules, which propagate by diffusion, modeled as Brownian motion [35]. The molecules transmitted by the TX node, and representing the signal to be delivered, are labeled as $S$, and those transmitted by the control node $\mathrm{RX}$, to encode the control messages, are labeled $R$. The Brownian motion is characterized by the diffusion coefficient, given by $D=\frac{K_{b} T}{6 \pi \eta r_{c}} . K_{b}$ is the Boltzmann constant, $T$ denotes the temperature expressed in Kelvins, $\eta$ is the viscosity of the medium, and $r_{c}$ is the radius of the considered molecules, i.e. $r_{c, r x}$ for type $R$ molecules and $r_{c, t x}$ for type $S$ molecules.

When a ligand molecule hits a free, compliant receptor with radius $r_{r}$, a bond is established. The bonding duration (or trafficking time) is modeled with an exponential random variable [32], with average value $T_{\text {traff }}$. A number $R$ of receptors $\left(R_{T X}\right.$ on the TX for $R$ molecules and $R_{R X}$ on the RX for $S$ ones) is distributed over the surface of each nanomachine.

Without any loss of generality, we use the protocol defined in [11], where the control signals are encoded in different patterns of bursts, with size $B_{R X}$ of $R$ molecules, by using the OOK modulation, and symbol duration $T_{S}$. The defined control messages are: START, encoded as 110, CONTROL, encoded as 10, and STOP, encoded as 111. For decoding the control bursts, the TX node adopts a threshold mechanism, with value $\zeta_{S}$. Motivations about the design choices of the control protocol can be found in [11], whereas values used for carrying out simulations are reported in Table 1. We stress that any other connection-oriented protocol can be used to the goal of this paper, and that the choice of adopting the one presented in [11] as underlying communication framework is due to the fact that, to the best of the authors' knowledge, it is the only one currently proposed in literature.

In realistic condition, since the distance between RX and TX is unknown, the TX "probes" the channel with an increasing rate of $S$ molecules in order to find the most suitable carrier release rate. This process is controlled by the RX, which sends throttling (CONTROL) messages when congestion is detected. Specifically, the TX nodes starts with a burst $B_{T X}=B_{0, T X}$ of $S$ molecules and continues increasing the transmission burst $B_{T X}$ by $B_{0, T X} S$ molecules each time interval $\Delta t$. Thus, the TX increases the release rate with a linear slope, until it receives either a STOP or a CONTROL message from the controller RX. Different reaction strategies to CONTROL messages at the TX node are presented in section 5.

At the RX site, connections are successfully established when $\zeta_{R T T} S$ molecules are received by a suitable time and, at the same time, also the round time time (RTT) of the communication path is estimated. The instant when the RTT is estimated and the connection is set up is denoted $t_{\zeta_{R T T}}$. The estimation of the RTT is equal to the time elapsed since the transmission of the final burst of the START signal from the RX to the time instant $t_{\zeta_{R T T}}$. Since $t_{\zeta_{R T T}}$, the RX starts monitoring the total amount of received $S$ molecules 
$N(t)$, updated with a period equal to $T_{w}$. The reception rate of the $S$ molecules is denoted $\lambda_{a}(t)$, thus $N(t)=\int_{0}^{t} \lambda_{a}(t)$. However, when a molecule hits a compliant receptor already busy in another bond, it cannot establish a bond with it and we say that it is "rejected", as in an already full queuing system [8,9]. The rate of such rejections is denoted $\lambda_{r}(t)$. When a rejection happens, the ligand molecule is bounced back as a result of a partially inelastic collision. In [33], an alternative model to re-position a detached molecule that breaks a bond is presented.

The transmission is ended by the RX by sending a STOP message when either it estimates that the target amount of absorbed molecules $\zeta_{\text {stop }}$ has been already reached (i.e. $N(t) \geq \zeta_{\text {stop }}$ ) or it will be reached in the next few seconds by issuing a STOP message (i.e. $N_{\text {stop }}(t) \geq \zeta_{\text {stop }}$ ).

\section{DETECTION OF CONGESTION}

Intuitively, the RX can estimate that congestion conditions around the target are arising if the estimated absorption rate deviates from its expected behavior. Since the law used by the TX to transmit the signal is known by the RX (i.e. a linear increase by $B_{0, T X}$ of the burst size $B_{T X}$ each $\Delta t$ seconds), it is also known the expected assimilation profile at the RX, since the concentration of molecules around the RX linearly depends on the number transmitted ones. In addition, the authors of [29] have proved that upon transmitting a burst of $Q$ molecules, the number of received molecules for large times is well approximated by

$$
A_{R X}\left(R_{R X}, d, Q\right)=\frac{r_{R X}}{d} \frac{R_{R X} r_{r, r x}}{R_{R X} r_{r, r x}+\pi r_{R X}} .
$$

We stress that any other transmission law would work. The only requirement is that the receiver has to be aware of it. Since molecular communications are characterized by high latency and high response times, we keep the linear increase, as in [11], to cope with slow reaction rates. The idea is to estimate the assimilation curve at run-time, and to detect congestion when a significant decrease with respect to the expected value is observed. Such a decrease has to be large enough to avoid taking natural fluctuations as congestion due to noise sources $[30,36]$, and enough small to be early in detecting the phenomenon. In addition, since in general the estimation of a rate value is often noisy, we prefer to take our decisions on the overall number of assimilations (i.e. the overall stimulus received, which is the integral of the rate profile), which we will expect quadratic. Thus, the number of assimilations can be expressed as

$$
N(t)=N_{0}+a\left(t-t_{0}\right)^{2},
$$

where $N_{0}$ and $t_{0}$ are the initial offset values in terms of number of $S$ molecules and relevant time. In our algorithm, initially $N_{0}=\zeta_{R T T}$ and $t_{0}=t_{\zeta_{R T T}}$. What is missing is the estimate of the parameter $a$ of the assimilation curve, which may depend on different factors, such as trafficking time, number of receptors, distance between TX and RX, unexpected attenuation phenomena, or presence of noise sources. We propose a least squares estimate of $a$, that in the considered case turns out to be quite simple:

$$
a=\frac{\sum_{j=1}^{N_{s}}\left(N(t)-N_{0}\right)\left(t-t_{\zeta_{R T T}}\right)^{2}}{\sum_{j=1}^{N_{s}}\left(t-t_{\zeta_{R T T}}\right)^{4}},
$$

where the number of the samples is equal to $N_{s}=\left\lfloor p R T T / T_{w}\right\rfloor$, and $p$ is the fraction of the initial RTT duration used to carry out the estimation. We selected a variable duration of the estimation time, since it is more adaptive to the working context. In particular, binding it to the RTT value allows taking into account the distance among the TX and RX. We have verified numerically that, up to RTT seconds after RTT estimation, congestion has still not occurred. The overall machinery, including all operations carried out by the RX, is illustrated in Fig. 1. As shown in the figure, the RX takes a number of samples of $a$, to implement (3), and only when $a$ is estimated it starts detecting possible congestion conditions.

As for this last point, we make use of two different functions, $N_{\text {exp }}(t)$ and $N_{\text {stop }}(t)$, to determine congestion and stop conditions. The first is equal to

$$
N_{\text {exp }}(t)=N_{0}+\beta a\left(t-t_{0}\right)^{2},
$$

where $\beta$ is the tolerance coefficient used to discriminate between congestion condition and random fluctuation of absorbed molecules, whereas the offset values depend on the selected throttling rate algorithm. They are function of the values $N_{c}, t_{\text {prev }}$, and $t_{c}$ estimated as shown in Fig. 1, and will be illustrated in the next section. As for the stop condition, the relevant function is equal to

$$
N_{\text {stop }}(t)=N(t)+2 a\left(t-t_{0}\right)\left((\|S T O P\|-1) T_{S}+R T T\right),
$$

which represents a linearization of the estimated curve (2) at time $t$, in order to take into account possible overload conditions occurring during the transmission of the STOP signal. The quantity $\|\cdot\|$ represents the number of symbols of a given sequence. The interval $t_{\text {lock }}-t$ represents the time needed to transmit the CONTROL message. During this interval all control operations at RX are frozen, since it cannot transmit anything until the control sequence has ended.

We remark that the law used here has the objective of an early congestion detection, differently from the one sketched in [11], which is less robust and designed to detect only heavy overload conditions. A further remark is that, even choosing an optimal rate in advance and keeping it constant for all the transmission time is tricky, since the actual distance among nanomachines is difficult to estimate precisely. Also the number of receptors is hardly known in advance, especially in bio-hybrid nodes [2], since it may be increased or decreased at runtime depending on the internal processing of the received stimulus, a process known as upregulation and downregulation, respectively [37]. Finally, the presence of inhibitory molecules in the surrounding environment [38] or of any other absorbing nodes [39] would alter the expected number of assimilated particles as well. Instead, the proposed adaptive approach learns the assimilation curve blindly with respect to all those parameters, thus largely simplifying the system design.

\section{CONGESTION CONTROL SCHEME}

In this section, we review four rate control algorithms, which can be triggered by the reception of a negative feedback (CONTROL message) sent by the RX. For each of them, we illustrate the new value of the rate upon feedback decoding, and also all the operation carried out at the RX in order to re-align with the new, expected assimilation profile.

\subsection{Algorithm "Restart"}

This algorithm is inspired by TCP Tahoe. However, since the slow-start method of the TCP would be to unfeasible in this scenario due to the absence of positive feedbacks, we will start directly with a scheme recalling the congestion avoidance. Specifically, when the CONTROL message is received, the transmission burst is reset to 1 molecule, that is $B_{T X}=B_{0, T X}$. At the RX side, this implies that the parabolic estimation restarts from the minimum of the parabola. In more detail, the effect of the control message will 


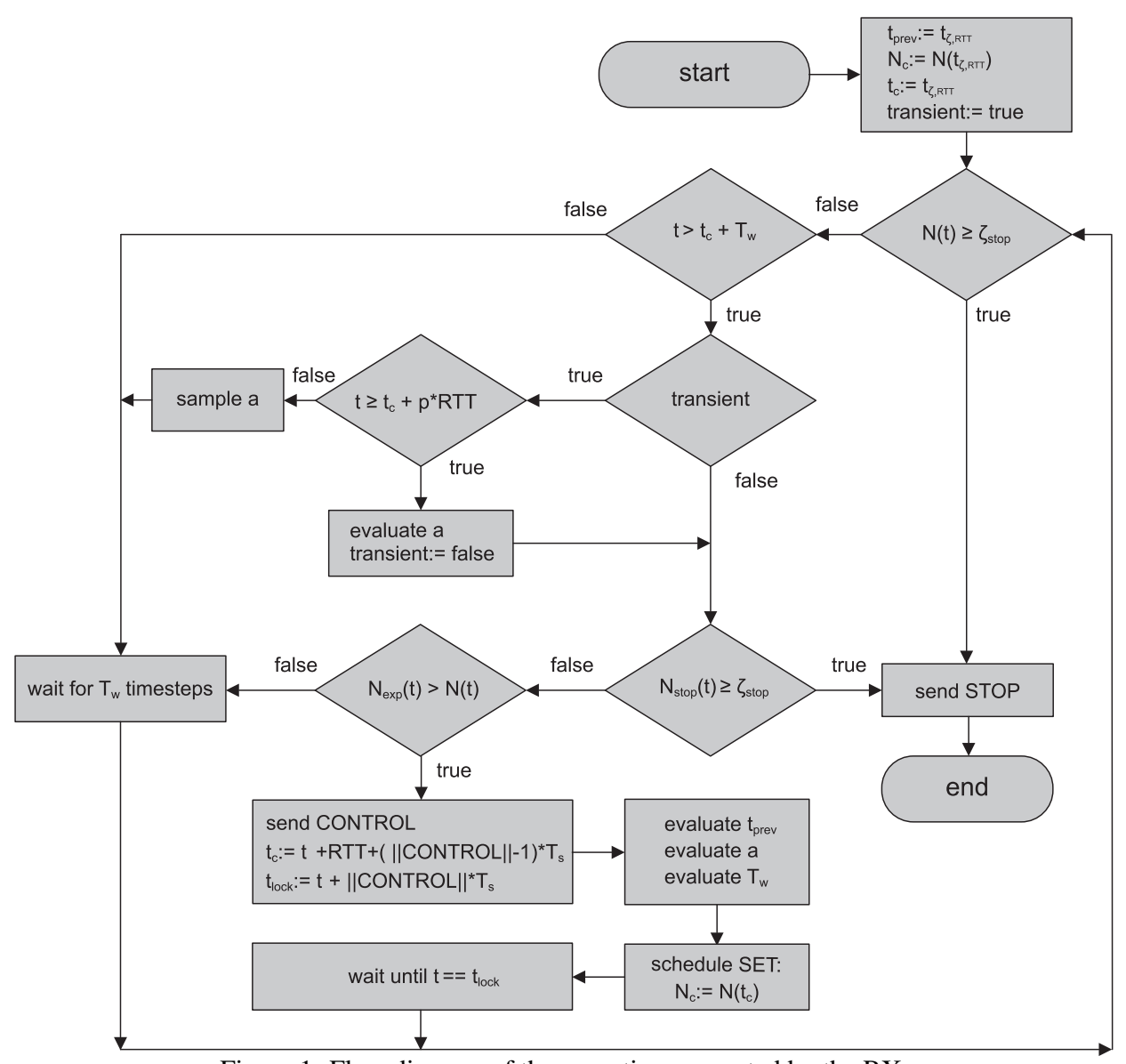

Figure 1: Flow diagram of the operations executed by the RX.

be visible at the RX at time $t_{c}=t+(\|C O N T R O L\|-1) T_{S}+R T T$ defined in Fig. 1 and thus $t_{0}=t_{c}$. The new offset will be estimated as the number of absorbed molecules at that time, that is $N_{0}=N_{c}=N\left(t_{c}\right)$. The value of $t_{c}$ takes into account the duration of transmission of control message, and the RTT time (see definition in section 3). Note that $t_{\text {prev }}$ in Fig. 1 is not used here. The rationale of this algorithm is to allow decongesting the RX, by restarting transmission from the beginning.

\subsection{Algorithm "Restart - Double"}

This variant, in addition to performing the same operation of the algorithm "Restart", it doubles the transmission time $\Delta t \leftarrow 2 \times \Delta t$. As a consequence, we also double the observation time $T_{w} \leftarrow 2 \times$ $T_{w}$ at the receiver, which has to be larger than the transmission time in order to correctly estimate the parabolic profile. The values of $N_{0}$ and $t_{0}$ are updated as in the previous case, but doubling the transmission time has an additional effect on the parameter $a$, which has to be updated accordingly. In particular, if in a time interval $T$ the number of transmission bursts are $n_{T}=T / \Delta t$, starting from $B_{0, T X}$, then the number of transmitted molecules is simply $N_{T}=n_{T}\left(n_{T}+1\right) / 2$. When the value of $\Delta t$ is doubled the number of transmitted molecules in the same interval are approximately decreased by a factor 4 , since

$$
N_{T}=\sum_{i=1}^{n_{T} / 2} i=\frac{n_{T}}{2} \frac{\left(n_{T} / 2+1\right)}{2}=\frac{1}{4} \frac{n_{T}\left(n_{T}+2\right)}{2} \approx \frac{N_{T}}{4} .
$$

Clearly, this translates into updating $a \leftarrow a / 4$. The rationale of this algorithm is to allow a complete decongestion of the RX, not only restarting the transmission process, but also by slowing the increase of the transmission rate.

\subsection{Algorithm "Halve"}

This algorithm is presented in [11], and inspired by TCP Reno. In this algorithm, the transmission window at the TX is halved, and not restarted from 1 molecule. Let us consider the time $t_{c}$ at which the effect of this action is visible at RX. Since the transmission period has not changed, the values of $a$ and $T_{w}$ will remain unchanged. As for the time shift $t_{0}$, since the rate is perceived as halved at $t_{c}$ and the previous starting point was estimated at $t_{0}=t_{\text {prev }}=t_{\zeta_{R T T}}$, then it is shifted to

$$
t_{0}=\frac{t_{c}+t_{\text {prev }}}{2},
$$

and then the new value of $t_{\text {prev }}$ is set to $t_{\text {prev }}=t_{0}$. As for $N_{\text {exp }}(t)$, the value of $N_{c}$ summarizes not only the number of assimilations before the control action, but also the initial $\left(t_{c}-t_{0}\right)$ seconds of the new parabola, as seen above. This means that

$$
N_{\text {exp }}(t)=N_{c}-\left(t_{c}-t_{0}\right)^{2}+\beta a\left(t-t_{0}\right)^{2},
$$

and thus $N_{0}=N_{c}-\left(t_{c}-t_{0}\right)^{2}$.

\subsection{Algorithm "Halve - Double"}

This last scheme is a variant of the "Halve" one, in which, similarly to "Restart - Double", upon receiving the CONTROL message, the TX node doubles the transmission period $\Delta t$. This implies that $\Delta t \leftarrow 2 \times \Delta t, T_{w} \leftarrow 2 \times T_{w}$, and $a \leftarrow a / 4$. As for $t_{0}$, the same 
Table 1: Simulation parameters

\begin{tabular}{l|l|l} 
Symbol & Description & Value \\
\hline$d t$ & Simulation time step & $20 \mu \mathrm{s}$ \\
$T$ & Temperature & $310 \mathrm{~K}$ \\
$e$ & Coefficient of restitution (partially inelastic collisions) & 0.9 \\
$\eta$ & Viscosity & $0.0011 \mathrm{Kg} \times(\mathrm{ms})^{-1}$ \\
$\beta$ & Tolerance factor & 0.95 \\
$T_{S}$ & Symbol time & $10 \mathrm{~s}[11]$ \\
$r_{R X}$ & Radius node RX & $2.5 \mu \mathrm{m}$ \\
$r_{T X}$ & Radius node TX & $2.5 \mu \mathrm{m}$ \\
$R_{R X}$ & Amount of surface receptors (node RX) & 10000 \\
$R_{T X}$ & Amount of surface receptors (node TX) & 10000 \\
$r_{c, r x}$ & Radius emitted molecules (type $R$ ) & $3.5 \mathrm{~nm}$ \\
$r_{c, t x}$ & Radius emitted molecules (type $S$ ) & $1.75 \mathrm{~nm}$ \\
$r_{r, r x}$ & Receptor radius (RX), type $S$ & $4 \mathrm{~nm}$ \\
$r_{r, t x}$ & Receptor radius (TX), type $R$ & $8 \mathrm{~nm}$ \\
$T_{t r a f f}$ & Trafficking time & $4 \mathrm{~s}$ \\
$\zeta_{S}$ & Assimilation threshold (TX) & $100 R$ molecules \\
$\Delta t$ & Emission time (TX) & $60 \mathrm{~ms}$ \\
$\zeta_{\text {stop }}$ & Assimilation threshold for STOP signal (RX) & 100000 molecules \\
$S T A R T$ & Signal pattern: START & 110 \\
$C O N T R O L$ & Signal pattern: CONTROL & 10 \\
$S T O P$ & Signal pattern: STOP & 111 \\
$d$ & Simulated distance & $26.5 \mu m$ \\
$B_{R X}$ & Burst for control signals (RX) & $2000 R$ molecules \\
$B_{0, T X}$ & Initial burst (TX) & $1 \mathrm{~S}$ molecule \\
$\zeta_{R T T}$ & Threshold for RTT estimation (RX) & $5 \mathrm{~S}$ molecules \\
$T_{w}$ & Observation window default value (RX) & $200 \mathrm{~ms}$ \\
$p$ & Fraction of RTT used for $a$ estimation & 0.5 \\
& &
\end{tabular}

considerations done for the case "Halve" hold, but, in addition, we have to consider that the transmission rate increase is halved. Thus, according to the new transmission profile, in the time interval $\left(t_{c}-t_{0}\right)$, the TX would have transmitted exactly half the number of bursts with respect to the previous configuration, and thus it simply results that $t_{0}$ remain unchanged $\left(t_{0}=t_{\zeta_{R T T}}\right)$. As for $N_{\text {exp }}(t)$, it has the same value of (8), but with the updated $a$ value.

\section{PERFORMANCE EVALUATION}

The performance evaluation of the system has been carried out by using the BiNS2 simulator. The main simulation parameters, together with their descriptions and values, are reported in Table 1.

We first performed a joint preliminary analysis in order to select the values of $p$ parameters that allows obtaining a reliable estimation of $a$. For space limitations, we do not show it in details. We found that, for very low values of the parameter $p$, the estimation of $a$ is quite unreliable, due to the low number of samples. As the value of $p$ approaches 0.5 , the estimation gets stable, and the decrease rate with $p$ becomes less significant. Thus, in order to get a reliable estimation, values in the order of 0.5-1 are recommended. In addition, we evaluated also the impact of different $T_{\text {traff }}$ and $d$ values on the estimation of $a$. We found that binding the estimation of $a$ to the value of RTT by means of $p$ is reasonable since, when the distance increases, the $S$ molecules concentration decreases, thus larger observation times due to larger RTT values do not lead to congestion. Note that the value selected for the trafficking times is realistic, since [32] reports also larger ones, in the order of many tens of seconds. In this paper, we used $p=0.5$.

Now, let us consider the system performance in terms of delivery efficiency and delivery time. We show some figure reporting time varying quantities, all for the case $T_{\text {traff }}=4 \mathrm{~s}$ and $p=0.5$

Fig. 2 shows, for the four proposed algorithms, the values of assimilation $\left(\lambda_{a}\right)$ and rejection $\left(\lambda_{r}\right)$ rates, as a function of the simulation time. For all the schemes, it can be easily identified the time instant when $\lambda_{a}$ begins deviating from the linear increase, and thus congestion is detected.In any case, the values of the first peak for $\lambda_{a}$ are comparable, which are close to 1000 molecules/s, although some deviations occur due to the random nature of the assimilation process. In fact, the initial trigger of control messages is not uniform over time, since it depends on the initial, noisy estimation
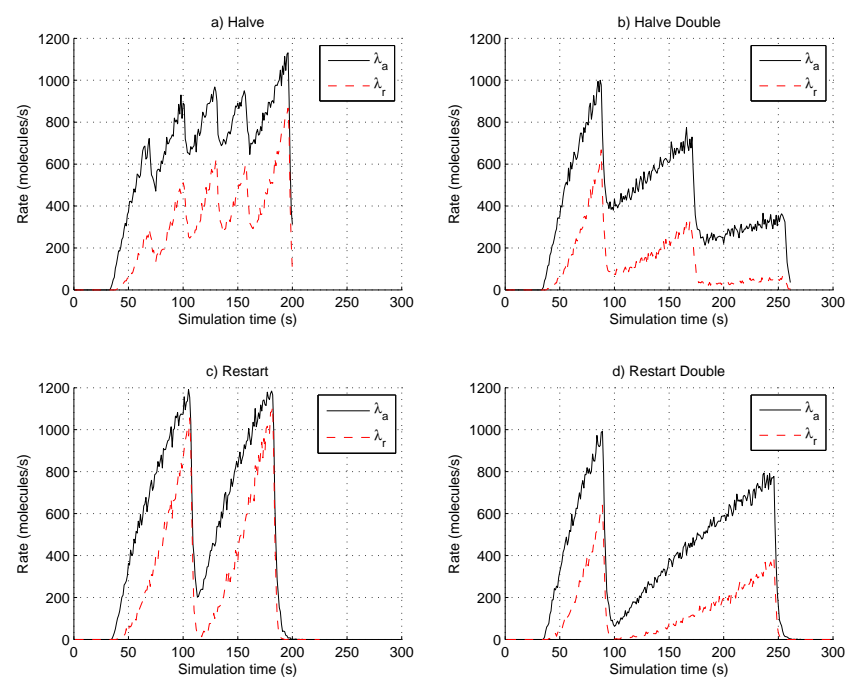

Figure 2: Assimilation $\left(\lambda_{a}\right)$ and rejection $\left(\lambda_{r}\right)$ rates as a function of time. Algorithms: a) Halve, b) Halve Double, c) Restat, and d) Restart Double.
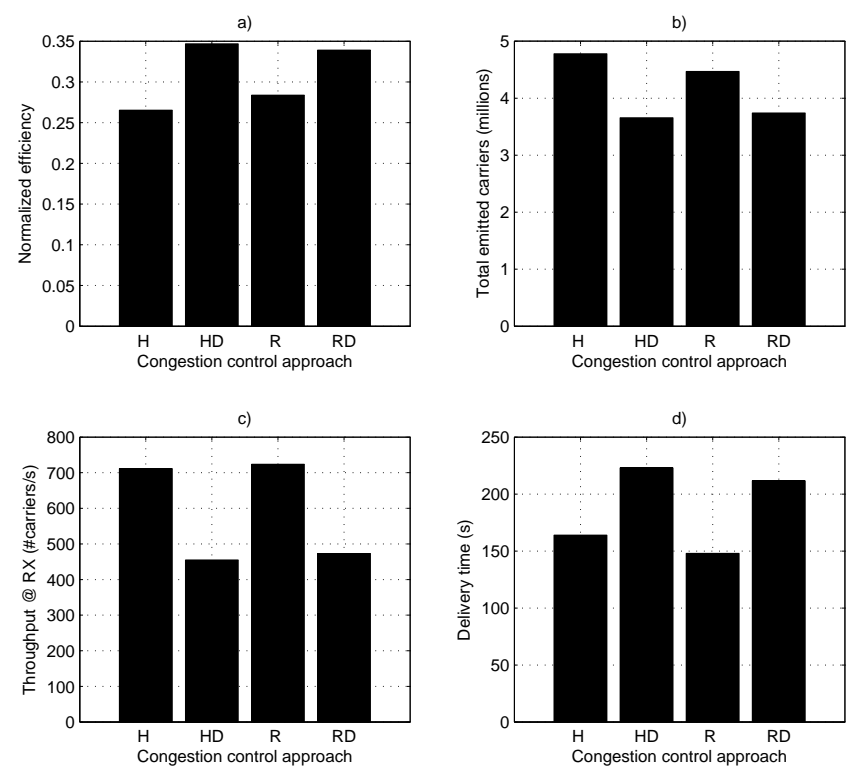

Figure 3: Global performance of the four congestion control schemes as a function of $T_{\text {traff }}$ and $p$ : a) delivery efficiency, b) total number of emitted carriers, c) receiver throughput, and d) delivery time. $\mathrm{H}$ means Halve, HD means Halve Double, R means Restart, and RD means Restart Double.

of $a$, and on the specific realization of the random arrival process. As for the Halve scheme (Fig. 2.a), it is evident a behavior which recalls the well known pattern of the TCP Reno. It is the control scheme that needs the transmission of the highest number of control messages. In the shown simulation, 4 control messages are sent. The net effect is that its assimilation rate, beyond an initial part which is clearly linear, evolves with a decreasing slope at the first $t_{c}$. In addition, this "continuous" transmission of control messages, in the specific simulation shown in the figure, causes some inefficiencies in meeting the target of $\zeta_{\text {stop }}$, due to the lock effect of these transmissions on other possible actions (see also Fig. 1).

Instead, in the Restart one (Fig. 2.c), it is evident a behavior similar to the TCP Tahoe, without the initial slow start effect. It 
is characterized by two aspects: the number of control messages is just one, since it is able to completely decongest the RX node, thanks to a complete re-initialization of the transmission burst $B_{T X}$, and the full shape of the linear absorption rate is well recognizable.

A common comment to the Double schemes is that, due to the increase (doubling) transmission intervals $\Delta t$, when the congestion is detected, the absolute values of the assimilation rate reaches always smaller peaks. In fact, the number of transmission bursts occurring during the time necessary to transmit, to propagate, and to decode the CONTROL message decreases. Thus, congestion is not exacerbated. This has a positive impact on the delivery efficiency. In addition, for the Restart Double scheme the same comments done for the Restart hold.

Finally, a common point to Halve and Restart schemes is that, thanks to a constant transmission interval $\Delta t$, they are able to complete the transmission in a shorter time.

Fig. 3 shows a number of performance metrics. Fig 3.a shows the delivery efficiency, defined as $\zeta_{\text {stop }} / A_{R X}\left(R_{R X}, d, N_{T}\right)$, where $N_{T}$ is the total number of emitted molecules by TX, as in (6), and $A_{R X}$, defined in (1), is the maximum number of molecules that RX is able to assimilate in absence of congestion upon a transmission of $N_{T}$ molecules and with absorbing receptors (i.e. it is an upper bound). First, we observe that the efficiency values are always below $50 \%$. In particular, it ranges from 0.25 for the Halve scheme, to about 0.35 for Halve Double and Restart Double.

Now, let us delve into comparison between congestion control algorithms. The Halve approach is the worst performing one in terms of efficiency, since it is the most aggressive, and forces the system to be continuously on the edge of congestion. On the other hand, Double-based approaches are the most efficient, since, by relaxing the transmission rate, they allows completely decongesting the $\mathrm{RX}$, and thus improving its capability of absorbing the surrounding molecules. The number of emitted carriers, shown in Fig. 3.b, is the dual metric of the efficiency. Thus, the worst performing control solution is the Halve, which wastes a lot of molecules since it maintains the RX always close to saturation. Instead, Doublebased approaches have similar behavior, with the best performance. Fig. 3.c show the throughput at the receiver, and Fig. 3.d its dual counterpart, i.e. the delivery time. Clearly, the Halve approach is the best performing one, having the highest throughput and, consequently, the lowest delivery time. Obviously, Double-based approach is the worst performing ones with respect to throughput and delivery time. In particular, the Restart Double approach, which is slightly less aggressive than the Halve Double approach, has the lowest throughput and, consequently, the highest delivery time. Summarizing, if all the performance metrics are equally important we can conclude that the best trade-off is the Restart approach, which exhibits good values in all metrics, by balancing efficiency and throughput. However, in the simulation presented, the Restart approach has been tested in a favorable condition, since it reaches the target $\zeta_{\text {stop }}$ with the peak rate (see Fig. 2.c). Thus, in more general conditions, it could have slightly less good performance. The less convenient approach is instead the Restart Double, which has performance similar to Halve Double in terms of high efficiency, but worst performance in terms of throughput and delivery time.

\section{CONCLUSION}

In this paper, we have analyzed do methods for handling congestion in diffusion-based molecular communications. We have first proposed a simple yet effective algorithm to detect congestion in its early stages. The proposed algorithm can be easily implemented in a device with limited memory and computing capabilities, and thus fits the requirements of nanomachines. In addition, it needs mini- mal configuration, since it estimates at run-time the system behavior and adapts accordingly. Then, we have proposed and compared four different control actions, triggered by the negative feedback sent by the receiver nanomachine and implemented in the transmitter nanomachine. From our results, obtained by considering realistic large trafficking times, it emerges that the best control scheme is the so-called Restart one, which couples the ability to decongesting the receiver with the capacity to quickly recover towards sustained bit rates. An interesting option could be also an hybrid approach, able to relax the transmission rate by means of the so-called "Halve - Double" scheme until the need of control actions is not frequent, and then using again the "Halve" control algorithm to maintain an high throughput. Clearly, in order to implement such an hybrid scheme, it is necessary to reconsider the message encoding adopted here and previously proposed in [11], by augmenting the number of symbols and thus the round trip latency of control actions, or resorting to molecule shift keying modulation (MoSK [40]) and using two types of molecules when switching from "Halve - Double" to "Halve".

Our ongoing and future work includes the extension of the congestion control strategy in a multi-access environment, which includes multiple TX and RX nodes, the commands of which may interfere each other. Another issues worth of investigation is the introduction of moving nanomachines. In this case, the estimation of the assimilation profile should be repeated upon movement detection, which could be implemented by periodically repeating the estimation of the RTT with off-band signaling, which translates into the usage of an additional, different types of control molecules.

\section{ACKNOWLEDGMENTS}

This work is supported by the EU project H2020 FET Open CIRCLE (project No. 665564) and by the MolML project funded by University of Perugia.

\section{REFERENCES}

[1] S. K. Khaitan and J. D. McCalley. Design techniques and applications of cyberphysical systems: A survey. IEEE Systems Journal, 9(2):350-365, June 2015.

[2] Ian F. Akyildiz, Fernando Brunetti, and Cristina Blázquez. Nanonetworks: A new communication paradigm. Comput. Netw., 52(12):2260-2279, August 2008.

[3] T. Nakano, M.J. Moore, Fang Wei, A.V. Vasilakos, and Jianwei Shuai. Molecular communication and networking: Opportunities and challenges. IEEE Transactions on NanoBioscience, 11(2):135-148, 2012.

[4] V. Loscri, C. Marchal, N. Mitton, G. Fortino, and AV. Vasilakos. Security and privacy in molecular communication and networking: Opportunities and challenges. NanoBioscience, IEEE Transactions on, 13(3):198-207, Sept 2014.

[5] L. Felicetti, M. Femminella, G. Reali, and P. Lio. Applications of molecular communications to medicine: A survey. Nano Communication Networks, 7:27 - 45, 2016.

[6] I. F. Akyildiz, M. Pierobon, S. Balasubramaniam, and Y. Koucheryavy. The internet of bio-nano things. IEEE Communications Magazine, 53(3):32-40, March 2015.

[7] U.A. Chude-Okonkwo et al. Molecular communication model for targeted drug delivery in multiple disease sites with diversely expressed enzymes. IEEE Trans. on NanoBioscience, 15(3):230-245, April 2016.

[8] M. Femminella, G. Reali, and A. V. Vasilakos. A molecular communications model for drug delivery. IEEE Transactions 
on NanoBioscience, 14(8):935-945, Dec 2015.

[9] Luca Felicetti, Mauro Femminella, and Gianluca Reali. A simple and scalable receiver model in molecular communication systems. In Proceedings of the 3rd ACM International Conference on Nanoscale Computing and Communication, NANOCOM'16, pages 39:1-39:2, New York, NY, USA, 2016. ACM.

[10] Tadashi Nakano, Yutaka Okaie, and Athanasios V. Vasilakos. Transmission rate control for molecular communication among biological nanomachines. IEEE Journal on Selected Areas in Communications, 31(12, supplement), 2013.

[11] L. Felicetti, M. Femminella, G. Reali, T. Nakano, and Athanasion V. Vasilakos. TCP-like molecular communications. Selected Areas in Communications, IEEE Journal on, Dec. 2014.

[12] Luca Felicetti, Mauro Femminella, and Gianluca Reali. A simulation tool for nanoscale biological networks. Nano Communication Networks, 3(1):2-18, 2012.

[13] Luca Felicetti, Mauro Femminella, Gianluca Reali, Paolo Gresele, and Marco Malvestiti. Simulating an in vitro experiment on nanoscale communications by using bins 2 . Nano Communication Networks, 4(4):172 - 180, 2013.

[14] M.J. Moore, T. Suda, and K. Oiwa. Molecular communication: Modeling noise effects on information rate. IEEE Transactions on NanoBioscience, 8(2):169-180, 2009.

[15] T. Nakano, Y. Okaie, and Jian-Qin Liu. Channel model and capacity analysis of molecular communication with brownian motion. Communications Letters, IEEE, 16(6):797-800, 2012.

[16] M. S. Kuran, H. B. Yilmaz, T. Tugcu, and B. Ozerman. Energy model for communication via diffusion in nanonetworks. Nano Communication Networks, 1(2):86 95, 2010.

[17] S. Kadloor, R.S. Adve, and A.W. Eckford. Molecular communication using brownian motion with drift. IEEE Transactions on NanoBioscience, 11(2):89-99, June 2012.

[18] K. V. Srinivas, A.W. Eckford, and R.S. Adve. Molecular communication in fluid media: The additive inverse gaussian noise channel. IEEE Transactions on Information Theory, 58(7):4678-4692, 2012.

[19] T. Nakano and Jian-Qin Liu. Design and analysis of molecular relay channels: An information theoretic approach. IEEE Transactions on NanoBioscience, 9(3):213-221, 2010.

[20] T. Nakano and Jianwei Shuai. Repeater design and modeling for molecular communication networks. In IEEE INFOCOM Workshop, pages 501-506, 2011.

[21] N. Farsad, A.W. Eckford, and S. Hiyama. A markov chain channel model for active transport molecular communication. Signal Processing, IEEE Transactions on, 62(9):2424-2436, May 2014.

[22] A. Guney, B. Atakan, and O.B. Akan. Mobile ad hoc nanonetworks with collision-based molecular communication. IEEE Transactions on Mobile Computing, 11(3):353-366, March 2012.

[23] Baris Atakan and Ozgur B. Akan. On molecular multiple-access, broadcast, and relay channels in nanonetworks. In ICST BIONETICS 2010, 2010.

[24] Baris Atakan and Ozgur B. Akan. Deterministic capacity of information flow in molecular nanonetworks. Nano Communication Networks, 1(1):31 - 42, 2010.

[25] Mohammad Upal Mahfuz, Dimitrios Makrakis, and
Hussein T. Mouftah. On the characterization of binary concentration-encoded molecular communication in nanonetworks. Nano Communication Networks, 1(4):289 300, 2010.

[26] M. Pierobon and I.F. Akyildiz. A physical end-to-end model for molecular communication in nanonetworks. IEEE Journal on Selected Areas in Communications, 28(4):602-611, 2010.

[27] M. Pierobon and I.F. Akyildiz. Diffusion-based noise analysis for molecular communication in nanonetworks. IEEE Transactions on Signal Processing, 59(6):2532-2547, 2011.

[28] H.B. Yilmaz, A.C. Heren, T. Tugcu, and Chan-Byoung Chae. Three-dimensional channel characteristics for molecular communications with an absorbing receiver. Communications Letters, IEEE, 18(6):929-932, June 2014.

[29] A. Akkaya, H.B. Yilmaz, C. Chae, and T. Tugcu. Effect of receptor density and size on signal reception in molecular communication via diffusion with an absorbing receiver. Communications Letters, IEEE, 19(2):155-158, Feb 2015.

[30] M. Pierobon and I.F. Akyildiz. Noise analysis in ligand-binding reception for molecular communication in nanonetworks. IEEE Transactions on Signal Processing, 59(9):4168 -4182, September 2011.

[31] L. Felicetti, M. Femminella, G. Reali, J.N. Daigle, M. Malvestiti, and P. Gresele. Modeling CD40-based molecular communications in blood vessels. IEEE Transactions on NanoBioscience, 13(3):230-243, 2014.

[32] D.A. Lauffenburger and J.J. Linderman. Receptors: Models for Binding, Trafficking, and Signalling,. Oxford University Press, 1996.

[33] Y. Deng, A. Noel, M. Elkashlan, A. Nallanathan, and K. C. Cheung. Modeling and simulation of molecular communication systems with a reversible adsorption receiver. IEEE Transactions on Molecular, Biological and Multi-Scale Communications, 1(4):347-362, Dec 2015.

[34] Y. Lu, M. D. Higgins, A. Noel, M. S. Leeson, and Y. Chen. The effect of two receivers on broadcast molecular communication systems. IEEE Transactions on NanoBioscience, 2016.

[35] J. Philibert. One and a half century of diffusion: Fick, einstein, before and beyond. Diffusion Fundamamentals, 4:6.1-6.19, 2006.

[36] M. Pierobon and I.F. Akyildiz. Capacity of a Diffusion-Based Molecular Communication System With Channel Memory and Molecular Noise . IEEE Transactions on Information Theory, 59(2):942-954, February 2013.

[37] Joseph Feher. Quantitative Human Physiology, An Introduction. Academic Press, Boston, 2012.

[38] A Noel, K.C. Cheung, and R. Schober. Improving receiver performance of diffusive molecular communication with enzymes. NanoBioscience, IEEE Transactions on, 13(1):31-43, March 2014.

[39] Y. Okaie, T. Nakano, T. Hara, and S. Nishio. Distributing nanomachines for minimizing mean residence time of molecular signals in bionanosensor networks. Sensors Journal, IEEE, 14(1):218-227, Jan 2014.

[40] Hoda ShahMohammadian, Geoffrey G. Messier, and Sebastian Magierowski. Optimum receiver for molecule shift keying modulation in diffusion-based molecular communication channels. Nano Communication Networks, 3:183-195, 2012. 